\title{
Human mannose-binding protein carbohydrate recognition domain trimerizes through a triple $\alpha$-helical coiled-coil
}

\author{
S. Sheriff, C.Y. Chang and R.A.B. Ezekowitz \\ Nature Structural Biology 1, 789-794 (1994).
}

The following errors have been noted by the authors since publication:

p. 790, second column, third line from the bottom should read:

In rat MBP, Trp 105 and Phe 138 are replaced by Leu 98 and Leu $131, \ldots$

p. 792, Table 1, under Refinement, second line, should read:

Number of reflections

6244

6318

\section{The not-so-great escape}

\section{J. Erickson}

Nature Structural Biology 2, 523-529 (1995)

The section under the subheading Cleavage-site mutants (p. 526, starting the sixth line from the bottom of the page) should read:

This may be especially important where a mutant HIV PR becomes rate-limiting for virus replication. Recent evidence from in vitro studies reveals that mutations in gag polyprotein cleavage sites can synergize with I84V or A mutations in the protease to produce a virus with $10^{2}-10^{3}$-fold decreased sensitivity to an inhibitor (D. Lamarre et al., pers. comm.). One such gag mutation was identified as a change in the P1 residue of the P1/P6 cleavage site from a Leu to a Phe, which alters the cleavage site from Phe-Leu to Phe-Phe. A synthetic peptide containing the Phe-Phe cleavage site is cleaved at a higher catalytic efficiency by both mutant and wild-type HIV $\mathrm{PR}$ than the corresponding peptide with the wild-type sequence.

\section{Structure of the $\mathrm{Ca}^{2+}$-free GLA domain sheds light on membrane binding of blood coagulation proteins}

\section{Sunnerhagen, S. Forsén, A-M Hoffrén, T Drakenberg, O. Teleman and J. Stenflo Nature Structural Biology 2, 504-509.}

The following errors have been noted:

Page 505, Fig. 1. h-factor X, residues 34-40 should read: S D K T N $\gamma$ F

Page 505, Fig. 1. h-Factor IX, residues 34-40 should read: T $\gamma \mathrm{R} \mathrm{T} \mathrm{T} \gamma \mathrm{F}$

Page 506, first column, line 10 should read: Mutating any of Gla residues 7, 16, 20, 26 or 29 to Asp results in loss of membrane binding and/or biological activity $y^{22,23}$. 